\title{
Influence of noise and near-resonant perturbations on bifurcations in Josephson junctions
}

\author{
Svensmark, Henrik; Hansen, Jørn Bindslev; Pedersen, Niels Falsig
}

Published in:

Physical Review A

Link to article, DOI:

10.1103/PhysRevA.35.1457

Publication date:

1987

Document Version

Publisher's PDF, also known as Version of record

Link back to DTU Orbit

Citation (APA):

Svensmark, H., Hansen, J. B., \& Pedersen, N. F. (1987). Influence of noise and near-resonant perturbations on bifurcations in Josephson junctions. Physical Review A, 35(3), 1457-1459.

https://doi.org/10.1103/PhysRevA.35.1457

\section{General rights}

Copyright and moral rights for the publications made accessible in the public portal are retained by the authors and/or other copyright owners and it is a condition of accessing publications that users recognise and abide by the legal requirements associated with these rights.

- Users may download and print one copy of any publication from the public portal for the purpose of private study or research.

- You may not further distribute the material or use it for any profit-making activity or commercial gain

- You may freely distribute the URL identifying the publication in the public portal

If you believe that this document breaches copyright please contact us providing details, and we will remove access to the work immediately and investigate your claim 


\title{
Influence of noise and near-resonant perturbations on bifurcations in Josephson junctions
}

\author{
H. Svensmark, J. Bindslev Hansen, and N. F. Pedersen \\ Physics Laboratory I, The Technical University of Denmark, DK-2800 Lyngby, Denmark
}

(Received 27 October 1986)

\begin{abstract}
The stabilization of a nonlinear system against period-doubling bifurcations by means of a periodic perturbation has been investigated. With the Josephson junction as a model system, both numerical simulations (including noise) and measurements on Josephson tunnel junctions have been performed. The effect of the noise is found to be of fundamental importance.
\end{abstract}

The phenomenon of small signal amplification in bifurcating dynamical systems has recently received renewed attention. $^{1,2}$ The somewhat opposite but related problem is that of dynamic stabilization of a nonlinear system against period-doubling bifurcations by use of a periodic perturbation. ${ }^{3}$

To exemplify these phenomena the Duffing oscillator has been considered as a model system, ${ }^{1,3}$ but the concepts and methods are assumed to be generic and applicable to related nonlinear systems such as lasers, ${ }^{4}$ Josephson junctions, ${ }^{5}$ semiconductors, etc.

The starting point is a dynamical system which is driven at a frequency $\omega_{D} \cdot{ }^{1,2}$ At a particular value $\mu_{0}$ of a control parameter $\mu$ the system undergoes a period-doubling bifurcation to a limit cycle of frequency $\omega_{D} / 2$. A periodic perturbation with a frequency $\omega_{S}$ in the vicinity of $\omega_{0} / 2$ is shown to suppress the period-doubled limit cycle, ${ }^{3}$ giving rise to stable motion beyond $\mu_{0}$. Furthermore, the perturbation itself may be amplified when the control parameter is just below $\mu_{0}{ }^{1,2}$

The purpose of the present paper is twofold. First, we calculate numerically the influence of noise on the stabilization by the periodic signal; second, we investigate experimentally the phenomena in a real physical system with noise, the Josephson junction.

The microwave driven Josephson junction is described by the following equation: ${ }^{6}$

$\phi_{t t}+\alpha \phi_{t}+\sin \phi=A_{D} \sin \Omega_{D} t+A_{S} \sin \Omega_{S} t+\eta+n(t)$.

Here $\phi$ is the quantum-mechanical phase difference across the junction and $\alpha$ is a damping parameter $\left(\alpha=1 / \sqrt{\beta_{c}}\right.$, where $\beta_{c}$ is the McCumber parameter $\left.{ }^{6}\right) . A_{D}$ and $A_{S}$ are the driving and perturbing signal amplitudes normalized to the critical current of the junction $I_{0}$, and $\Omega_{D}$ and $\Omega_{S}$ are the corresponding frequencies normalized to the maximum plasma frequency $\omega_{p 0}$ of the junction. $\eta$ is the (normalized) dc bias current and $n(t)$ is a noise current to be specified below. We note that Eq. (1) also describes a driven damped pendulum.

In the numerical calculations described here, Eq. (1) is integrated using a second-order Runge-Kutta method with 32 points per period of the drive. 512 drive periods are integrated, and the first 256 are discarded to remove transients. The noise is assumed to be a white spectrum described by the parameter $\Gamma=2 e k_{B} T / \hbar I_{0}$, i.e., the ratio of the thermal energy $\left(k_{B} T\right)$ to the Josephson coupling ener- gy $(\hbar / 2 e) I_{0}$. Typical experimental values for Josephson junctions $\left(I_{0}=0.1-1 \mathrm{~mA}\right.$ and $\left.T=4 \mathrm{~K}\right)$ correspond to $\Gamma=10^{-3}$ to $10^{-4}$. In the calculations the white noise is constructed using a random number generator. Throughout the calculations the following values of the parameters were assumed: $\alpha=0.2, \Omega_{D}=1.6, A_{S}=0.01$, $\Omega_{S}=(127 / 256) 1.6\left(\right.$ close to $\left.\Omega_{D} / 2\right)$, and $A_{D}, \eta$, and $n(t)$ were varied.

Figure 1 illustrates for $A_{D}=0.98$ and $\Omega_{D}=1.6$ an example of the influence of noise and a stabilizing periodic signal on the period-doubling bifurcation. Here the (normalized) amplitude of the period-doubled signal is plotted versus the control parameter, which is the normalized bias current $\eta$. Curve a shows the amplitude of the perioddoubled signal in the absence of both signal and noise [ $A_{S}=0, n(t)=0$ ]. In curve b thermal noise (with $\left.\Gamma=10^{-4}\right)$ is included, but the stabilizing periodic signal is absent $\left(A_{S}=0\right)$. For $A_{S}=0$ the effect of noise is evidently only a very modest quantitative change (smoothing of the corners).

The effect of the stabilizing signal $\left[A_{S}=0.01\right.$, $\left.\Omega_{S}=(127 / 256) 1.6\right]$ in the absence of thermal noise

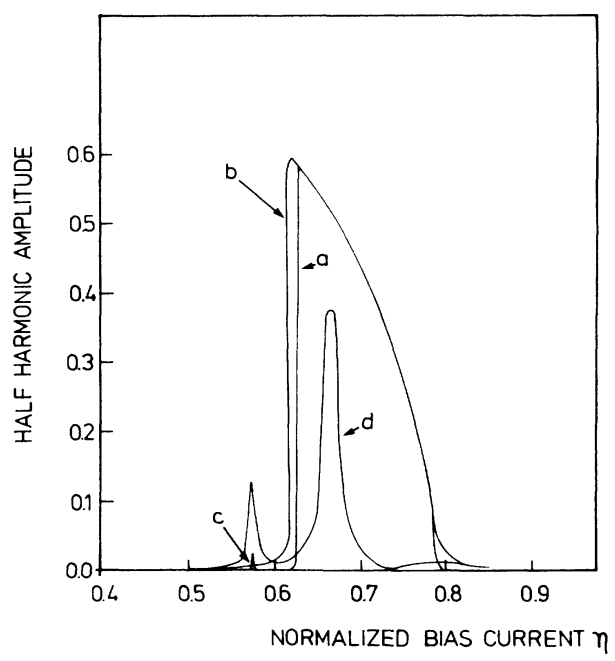

FIG. 1. Numerical calculation of half-harmonic amplitude for increasing bias current. $A_{D}=0.98, \Omega_{D}=1.6, \alpha=0.2$. (a) $\Gamma=0$, $A_{S}=0$; (b) $\Gamma=10^{-4}, A_{S}=0$; (c) $\Gamma=0, A_{S}=0.01, \Omega_{S}=(127 /$ 256)1.6; (d) $\Gamma=10^{-4}, A_{S}=0.01, \Omega_{S}=(127 / 256) 1.6$. 
$(\Gamma=0)$ is seen in $c$, where the period-doubled signal has been suppressed, confirming the ideas in Refs. 2 and 3. Notice, however, that c contains a small period-two component at $\eta=0.57$. This is outside the period-doubling region for increasing $\eta$, but at the end of this region for decreasing $\eta$ (hysteresis).

Finally, curve d of Fig. 1 shows the effect of including simultaneously the stabilizing signal $\left[A_{S}=0.01\right.$, $\left.\Omega_{S}=(127 / 256) 1.6\right]$ and the thermal noise $\left(\Gamma=10^{-4}\right)$. Now a signal at the period-doubled frequency $\Omega_{D} / 2$ reappears due to the effect of the noise. As a function of the dc bias three peaks are seen, of which the two outer peaks differ in nature from the middle one. Near the parameter values chosen, the center peak is much more dependent on the drive amplitude than the two outer peaks (see Fig. 4). The center peak behaves qualitatively as predicted in Refs. 2 and 3. For the two largest peaks the bifurcation point is exceeded. For all three peaks a noisy precursor is created near the period-doubling instability. ${ }^{7}$ We believe that the two outer peaks have not been observed previously. The apparent importance of the noise term is remarkable since in the nonstabilized spectrum (Fig. 1, curve a) the effects of noise were only minor and quantitative.

The qualitative behavior illustrated in Fig. 1 has been verified also for a number of other parameter values. The main lesson to learn from Fig. 1 is that for a meaningful comparison with experiments, the theory of the perioddoubling stabilization by means of a periodic perturbation must be extended to include the effects of noise.

To further illuminate the problems encountered in the numerical simulations of Fig. 1 we have carried out an experimental investigation of the effect of a small nearresonant perturbing microwave signal on the threshold for the half-harmonic generation (period-doubling bifurcation) in microwave-driven Josephson tunnel junctions. For these experiments we used $\mathrm{Nb}-\mathrm{NbO}_{x}-\mathrm{Pb}$ tunnel junctions of the overlap type. The junction parameters are listed in Table I.

The junction was incorporated into an inverted microstrip structure (Fig. 2) that was contained in a temperature-stabilized metal box with a large heat capacity. The box was placed in a vacuum can immersed in a liquid-helium bath. The junction was irradiated with a monochromatic microwave driving signal $I_{D} \sin \left(\omega_{D} t\right)$ from a source in the frequency range $16-20 \mathrm{GHz}$ (linewidth $<100 \mathrm{~Hz}$ ) coupled to the junction through a direct microstrip line seen in Fig. 2. The generated halfharmonic signal (in the vicinity of the junction plasma frequency) was coupled out through the same microwave line to a low-noise field-effect-transistor amplifier followed by a digital storage spectrum analyzer with a minimum resolution bandwidth of $100 \mathrm{~Hz}$. To avoid saturating the amplifier with the pump signal, low-pass filters were inserted

TABLE I. Junction parameters.

\begin{tabular}{lccrc}
\hline \hline Sample & $\begin{array}{c}\text { Dimensions } \\
(\mu \mathrm{m})^{2}\end{array}$ & $\begin{array}{c}I_{0}(4.2 \mathrm{~K}) \\
(\mathrm{mA})\end{array}$ & $\begin{array}{c}R_{N} \\
(\Omega)\end{array}$ & $\begin{array}{c}\omega_{p 0} / 2 \pi \\
(\mathrm{GHz})\end{array}$ \\
\hline S-5001 & $28 \times 32$ & 0.585 & 1.9 & 21.6 \\
S-5002 & $21 \times 31$ & 0.117 & 10.0 & 12.5 \\
\hline \hline
\end{tabular}

between the junction and the amplifier.

The perturbing periodic signal $I_{S} \sin \left(\omega_{S} t\right)$ from a second microwave source was coupled into the junction via the "L"-shaped antenna coupled microstrip line seen in Fig. 2. Its frequency was typically chosen to be $100 \mathrm{kHz}$ to $3 \mathrm{MHz}$ below the half-harmonic signal at $\Omega_{D} / 2$, i.e., the relative detuning $\delta=2\left(\Omega_{S}-\Omega_{D} / 2\right) / \Omega_{D}$ was in the range $10^{-5}$ to $3 \times 10^{-4}$. The linewidth of the perturbing signal was $2 \mathrm{kHz}$.

By measuring the suppression of the critical current as a function of microwave power and frequency from the two sources, we deduced that the microwave coupling around both the drive frequency and the half-harmonic frequency was strongly frequency dependent but always very weak. The measured dependence of the critical current of the junction as a function of microwave power was used to determine the amplitude of the microwave currents through the junction $A_{D}$ and $A_{S}{ }^{8}$

The effective noise temperature of the junction was also measured in situ by recording the noise-perturbed distribution of the critical current for a large number of switching events, i.e., the switching-point distribution for the junction (we typically collected the distribution of 25000 switching points in a low-noise data acquisition system). If we assumed a white noise spectrum such a measurement of the noise currents, primarily around the plasma frequency, yielded an effective noise temperature of about 12 $\mathrm{K}$ for our experimental setup (at a bath temperature of 4.2 K). ${ }^{8}$

The current and voltage leads connected to the junction were carefully shielded and filtered in order to minimize room-temperature noise and interference from external sources. The ambient magnetic field was reduced by a double $\mu$-metal shield. A small magnetic field could be applied in the plane of the junction from a $\mathrm{Cu}$ wire-wound solenoid. The entire experimental setup was enclosed in an rf-shielded room.

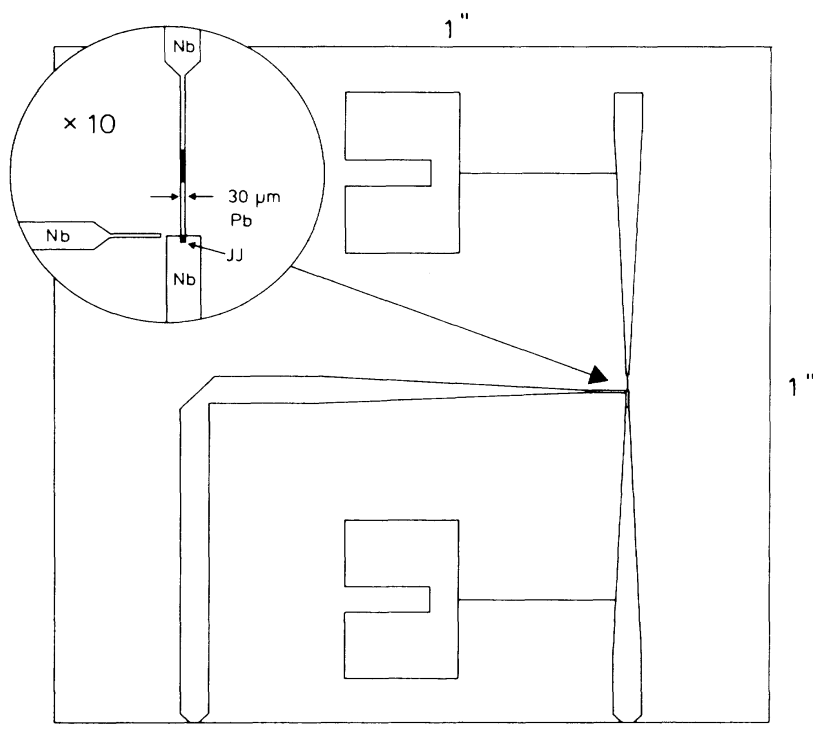

FIG. 2. Geometry of the sample and the microwave coupling circuit. 


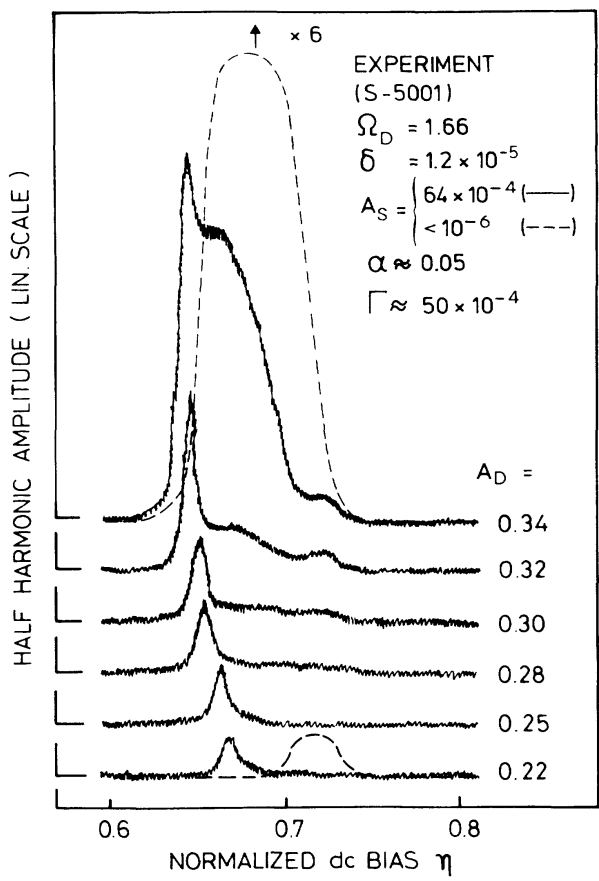

FIG. 3. Experimentally measured half-harmonic amplitude at $8.694 \mathrm{GHz}$. The changed parameter is the normalized drive amplitude $A_{D}$. The other parameters are shown in the figure.

The experimental investigation of the effect of the perturbing signal on the half-harmonic generation in the junction was carried out in the following way.

First, the dc-bias current, the magnetic field, and the temperature were chosen such that the resonance frequency of the junction was near $9 \mathrm{GHz}$ where the detection system was most sensitive. Second, with the perturbing signal amplitude set to zero the amplitude of the drive signal was adjusted to a value where the half-harmonic generation was observed. Recordings of the amplitude of the halfharmonic signal as a function of the dc bias were then taken (dashed curves in Fig. 3). Third, the perturbing signal was turned on and the suppression of the half-harmonic signal was investigated as a function of the frequency detuning $\delta$ and the signal amplitude $A_{S}$. A typical set of traces as function of dc bias for a number of values of $A_{D}$ (and constant $A_{S}$ ) is shown in Fig. 3 (full curves). The suppression of the half-harmonic generation in the junction did not happen abruptly as predicted in Refs. 2 and 3 for the noise-free case, but rather more continuously; even so, the stabilizing effect of the periodic perturbation is quite evident. The most interesting result of our experimental investigation is, however, the appearance of a new

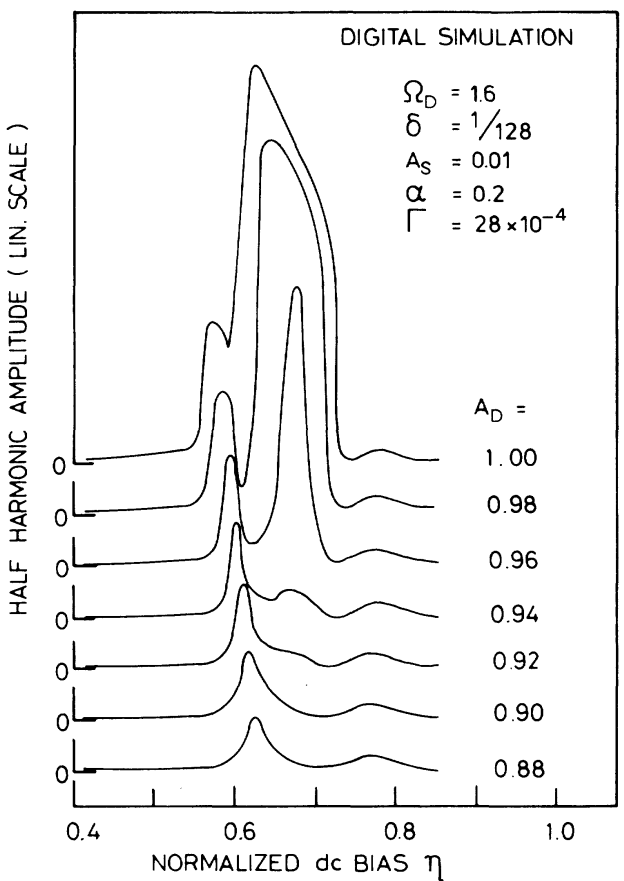

FIG. 4. Numerical calculation of half-harmonic amplitude. The changed parameter is $A_{D}$. The other parameters are shown in the figure.

instability region close to the original bifurcation point. This instability shows up as peaks in the half-harmonic amplitude traces in Fig. 3. We assume that this instability is of the same type as the one seen in the digital calculations (Fig. 1, curve d).

This assumption is supported by the series of numerically calculated curves presented in Fig. 4. Although the parameters are somewhat different from the experimental ones, the series of curves in Fig. 4 very clearly reproduces the gross features of the experiment in Fig. 3, in particular the appearance of multiple peaks. The main difference between the experimental and the numerical parameters lies in the value of the damping parameter $\alpha$. In order to save computer time, a rather high value of $\alpha(0.2)$ was chosen, whereas the experimental value was about 0.05 . The larger damping in the simulation is qualitatively consistent with the larger values of the detuning and the drive and the signal amplitudes found there. Work is in progress to carry out a more detailed quantitative comparison, and to understand the dynamical behavior more deeply.

Stimulating discussions with M. R. Samuelsen are gratefully acknowledged.
${ }^{1}$ Kurt Wiesenfeld and Bruce McNamara, Phys. Rev. Lett. 55, 13 (1985).

${ }^{2}$ Kurt Wiesenfeld and Bruce McNamara, Phys. Rev. A 33, 629 (1986).

${ }^{3}$ Paul Bryant and Kurt Wiesenfeld, Phys. Rev. A 33, 2525 (1986).

${ }^{4}$ B. Derighetti, M. Ravani, R. Stoop, P. F. Meier, E. Brun, and R. Badii, Phys. Rev. Lett. 17, 1746 (1985).
${ }^{5}$ Paul Bryant, Kurt Wiesenfeld, and Bruce McNamara (unpublished).

${ }^{6}$ For a good description of Josephson junction equations, see, for example, A. Barone and G. Paterno, Physics and Applications of the Josephson Effect (Wiley, New York, 1982).

${ }^{7}$ Kurt Wiesenfeld, J. Stat. Phys. 38, 1071 (1985).

${ }^{8}$ J. Bindslev Hansen et al., Appl. Phys. Lett. 49, 1744 (1986). 